\title{
Aplicación del aprendizaje basado en problemas para la enseñanza de la microbiología en estudiantes de Medicina
}

\author{
V. Lifschitz, A. Bobadilla, P. Esquivel, G. Giusiano, L. Merino
}

Introducción. El aprendizaje basado en problemas (ABP) es uno de los métodos de enseñanza-aprendizaje frecuentemente utilizado en las instituciones de educación superior en los últimos años, pero su aplicación aún no abarca todos los ámbitos de la Medicina. Objetivo. Evaluar el método del ABP en la enseñanza de la microbiología en comparación con los métodos tradicionales basados en talleres y seminarios. Sujetos y métodos. El estudio se realizó en la Cátedra de Microbiología, Parasitología e Inmunología de la Facultad de Medicina de la Universidad Nacional del Nordeste, Corrientes, Argentina. El total de alumnos de la asignatura se dividieron en un grupo control (a quienes se aplicó la enseñanza tradicional) y un grupo experimental (con quienes se trabajó con técnica de ABP). Los alumnos de ambos grupos fueron evaluados individualmente a través de un cuestionario de opciones múltiples y grupalmente mediante una encuesta de opinión. Resultados y conclusiones. No se observó diferencia significativa en cuanto a los conocimientos adquiridos en ambos grupos. El grupo de ABP mostró mayor compromiso y motivación para desarrollar la actividad asignada, pero esa diferencia no fue estadísticamente significativa.

Palabras clave. Aprendizaje basado en problemas. Educación médica. Microbiología.

\section{Application of the problem-based learning for the Microbiology teaching among Medicine students}

Introduction. The problem-based learning (PBL) is one of the teaching-learning methods more used in the institutions of higher education in recent years, but its application still does not cover all areas of Medicine. Aim. To compare the PBL with the traditional method based in seminars and workshops in the Microbiology teaching. Subjects and methods. The study was carried out in the subject of Microbiology, Immunology and Parasitology, Faculty of Medicine, Universidad Nacional del Nordeste, Corrientes, Argentina. Students were divided into a control group (in which the traditional teaching methods were applied) and an experimental group (in which the PBL was applied). Students were individually evaluated by a multiple choice questionnaire. One opinion survey was applied in groups. Results and conclusions. No significant difference was observed between the groups in terms of knowledge gained. The PBL group showed greater commitment and motivation to develop the assigned activity, although without statistically significant differences.

Key words. Medical education. Microbiology. Problem-based learning.

\section{Introducción}

Los nuevos papeles del profesor y del alumno implican cambios en las formas tradicionales de enseñar y aprender; en este contexto están adquiriendo importancia las metodologías docentes caracterizadas por el papel protagonista del estudiante en el reto de aprender [1].

$\mathrm{El}$ aprendizaje basado en problemas (ABP) es uno de los métodos de enseñanza-aprendizaje más utilizado en las instituciones de educación superior en los últimos años, pero su aplicación en la Facultad de Medicina de la Universidad Nacional del Nordeste es aún incipiente.

Esta metodología posee la propiedad de estar centrada en el alumno, propiciándose la reflexión crítica y el aprendizaje para toda la vida y es una
Cátedra de Microbiología, Parasitología e Inmunología. Carrera de Medicina. Facultad de Medicina. Universidad Nacional del Nordeste. Corrientes, Argentina.

Correspondencia Dr. Luis A. Merino. Cátedra de Microbiología, Parasitología e Inmunología Carrera de Medicina. Facultad de Medicina. Universidad Nacional del Nordeste. Sargento Cabral, 2001. 3400

Corrientes, Argentina.

E-mail catmicromed@gmail.com 
Tabla I. Distribución de las puntuaciones obtenidas en la evaluación de conocimientos en ambos grupos de estudio.

\begin{tabular}{lccc}
\hline & Grupo ABP & Control & $p$ \\
\hline N.o de alumnos no aprobados (0-3 puntos) & 28 & 21 & $0,68^{\text {a }}$ \\
\hline N.o de alumnos aprobados (4-10 puntos) & 71 & 69 & $0,54^{\text {b }}$ \\
\hline Promedio de calificaciones en la totalidad de los alumnos & 4,53 & 4,39 & $0,34^{\text {b }}$ \\
\hline Promedio de calificaciones entre alumnos aprobados & 5,15 & 4,96 & \\
\hline
\end{tabular}

ABP: aprendizaje basado en problemas. ${ }^{a}$ Calculado mediante chi al cuadrado; ${ }^{b}$ Calculado mediante $t$ de Student.

manera efectiva de educación médica basada en principios del aprendizaje de adultos [2].

El ABP constituye una metodología de aprendizaje en la cual el punto de partida es un problema o situación que permite al estudiante identificar necesidades para comprender mejor ese problema/situación, identificar principios que sustentan el conocimiento y cumplir objetivos de aprendizaje relacionados con cada porción del programa educacional [3]. Esto es así debido a que se considera que el aprendizaje significativo se produce sólo como resultado de la interacción entre la nueva información y la ya existente en las estructuras cognitivas del aprendizaje [4].

El objetivo de esta experiencia fue comparar los resultados obtenidos en la enseñanza de la microbiología mediante el ABP y el método tradicional.

\section{Sujetos y métodos}

La experiencia se desarrolló en el Área de Microbiología e Inmunología de la Cátedra de Microbiología, Parasitología e Inmunología de la Facultad de Medicina de la Universidad Nacional del Nordeste.

La asignatura se ubicó en el primer semestre del segundo año del plan de estudios vigente y poseía una matrícula de 189 alumnos con una edad promedio de 19 años. Con el fin de comparar los resultados obtenidos se formaron dos grupos cuyos integrantes fueron seleccionados en base a una lista ordenada alfabéticamente.

El grupo control estuvo formado por los primeros 90 alumnos de la lista, quienes cursaron la asignatura siguiendo el esquema tradicional (talleres y seminarios). Estaba conformado por 34 varones (38\%) y 56 mujeres (62\%), y a su vez se dividió en seis subgrupos de trabajo. Durante cuatro clases semanales, los alumnos participaron de actividades de seminarios y talleres según la forma tradicional de dictado de la asignatura.

La técnica del ABP se aplicó a un segundo grupo de 99 alumnos, integrado por un $38 \%$ de varones y un $62 \%$ de mujeres, distribuidos a su vez en 10 subgrupos de trabajo. La estrategia de ABP se desarrolló en cuatro sesiones semanales de 120 minutos cada una. El problema presentado se diseñó a partir de los resultados de un trabajo de investigación en el área de la microbiología clínica [5], el cual se adecuaba para generar múltiples hipótesis, estimular el pensamiento creativo, requerir de conocimientos previos, dirigir al estudiante hacia objetivos curriculares definidos, estimular la búsqueda de información y no precisar niveles excepcionales de conocimientos clínicos o razonamientos muy complicados [2].

En ambos grupos, los subgrupos se formaron por empatía entre los alumnos. A los siete días de finalizado el curso ordinario de la asignatura, se realizó la evaluación de los conocimientos adquiridos aplicando, sobre la totalidad de los alumnos, un cuestionario de opciones múltiples compuesto de 40 preguntas, con cinco distractores cada una. El cuestionario se consideró apro- 
Tabla II. Resultados de la encuesta de opinión mediante autoevaluación grupal.

\begin{tabular}{|c|c|c|c|}
\hline & $\begin{array}{c}\text { Grupos ABP } \\
10 \text { subgrupos }\end{array}$ & $\begin{array}{c}\text { Control } \\
6 \text { subgrupos }\end{array}$ & $p^{a}$ \\
\hline Motivación & & & 0,73 \\
\hline Pobre & $1(10 \%)$ & $1(17 \%)$ & \\
\hline Competente & $7(70 \%)$ & $3(50 \%)$ & \\
\hline Sobresaliente & $2(20 \%)$ & $2(33 \%)$ & \\
\hline Relaciones interpersonales & & & 0,41 \\
\hline Pobre & $0(0 \%)$ & $1(17 \%)$ & \\
\hline Competente & $4(40 \%)$ & $2(33 \%)$ & \\
\hline Sobresaliente & $6(60 \%)$ & $3(50 \%)$ & \\
\hline Actividad grupal & & & 0,38 \\
\hline Pobre & $0(0 \%)$ & $1(17 \%)$ & \\
\hline Competente & $5(50 \%)$ & $2(33 \%)$ & \\
\hline Sobresaliente & $5(50 \%)$ & $3(50 \%)$ & \\
\hline Habilidades para la resolución de problemas & & & 0,60 \\
\hline Pobre & $0(0 \%)$ & $0(0 \%)$ & \\
\hline Competente & $4(40 \%)$ & $4(67 \%)$ & \\
\hline Sobresaliente & $6(60 \%)$ & $2(33 \%)$ & \\
\hline
\end{tabular}

ABP: aprendizaje basado en problemas. ${ }^{2}$ Calculado mediante chi al cuadrado.

bado con una calificación de 4. Adicionalmente, se llevó a cabo una autoevaluación en forma grupal con el objeto de estimar motivación, relaciones interpersonales, actividad grupal y habilidades para la resolución de problemas, aplicando para ello la escala de Likert con tres categorías (pobre, competente y sobresaliente) [6].

Las diferencias entre los grupos control y experimental se evaluaron mediante las pruebas de $t$ de Student para las medias de calificaciones y de chi al cuadrado para el resto de las variables, considerándose estadísticamente significativo un valor de $p<0,05$.

\section{Resultados}

Los resultados obtenidos con el cuestionario individual se muestran en la tabla I, de la cual se 
desprende que no existió diferencia estadísticamente significativa entre el total de aprobados y no aprobados de ambos grupos. Igual consideración puede hacerse entre los promedios de calificaciones obtenidos en cada categoría, lo que evidencia que los conocimientos adquiridos fueron similares entre el grupo control y el experimental.

Los resultados de la encuesta de opinión aplicada sobre ambos grupos de alumnos se muestran en la tabla II, en la que se aprecia que aunque existieron diferencias entre ambos, éstas no fueron estadísticamente significativas.

\section{Discusión}

Se ha considerado que lo esencial del ABP es el aprendizaje autodirigido en el cual el estudiante aprende de forma autónoma; esto significa un cambio fundamental en el rol del docente que debe facilitar este proceso [7].

El análisis de los resultados de la evaluación de conocimientos adquiridos, realizada mediante un examen escrito común aplicado simultáneamente a los estudiantes de ambos grupos, permite concluir que el rendimiento obtenido por los alumnos de ABP es comparable al alcanzado mediante la enseñanza tradicional.

Estos resultados coinciden con otros metaanálisis de experiencias con $\mathrm{ABP}$, en los cuales la dimensión del efecto estadístico no fue significativamente diferente entre estudiantes que realizaron programas de ciencias básicas utilizando ABP y estudiantes que utilizaron docencia tradicional, siempre que se aplique la metodología de opciones múltiples [8-11].

Por otro lado, el análisis de la encuesta de opinión revela que la técnica de $\mathrm{ABP}$ se encuentra mejor calificada que los seminarios tradicionales como estrategia motivadora. El ABP también fue superior en los ítems de relaciones interpersonales, actividad grupal y habilidad de resolución de problemas. El compromiso con la actividad planteada fue superior en el grupo de ABP, según la observación de los docentes.

Se observó, al igual que en experiencias similares publicadas previamente [7], un desarrollo de habilidades deseables para su futura vida profesional, como acercamiento al pensamiento crítico, reconocimiento de necesidades de aprendi- zaje, incremento de habilidades para la búsqueda de información y su selección y valoración, acercamiento al trabajo cooperativo, autocrítica y responsabilidad.

$\mathrm{Al}$ enfrentarse a la resolución de una situación real, los estudiantes encontraron sentido a su aprendizaje, motivándose en la adquisición de un conocimiento significativo, lo cual los estimula y alienta.

No puede emitirse una opinión acerca de la capacidad del uso de los conocimientos, ya que esta habilidad no se evaluó en la presente circunstancia; no obstante, según Prince et al, el ABP prepara mejor para la aplicación del conocimiento que otros métodos [12].

La integración de las ciencias básicas y las ciencias clínicas ha sido propuesta como una importante necesidad educacional por diferentes grupos de expertos, propiciándose la utilización de metodologías innovadoras, en especial el ABP, como una de las fórmulas que más contribuye a lograr tal objetivo [8].

En conclusión, en base a los resultados obtenidos, puede afirmarse que el ABP constituye una opción más en el proceso de enseñanza-aprendizaje de la microbiología, en la que los alumnos se sienten más involucrados que en otras estrategias educativas.

\section{Bibliografía}

1. Escribano A, Del Valle A. El aprendizaje basado en problemas: una propuesta metodológica en educación superior. 1 ed. Madrid: Narcea; 2008.

2. Wood D. ABC of learning and teaching in Medicine. Problem based learning. BMJ 2003; 326: 328-30.

3. Branda LA. El aprendizaje basado en problemas en la formación en Ciencias de la Salud. In Alameda-Cuesta $\mathrm{C}$, ed. El aprendizaje basado en problemas: una herramienta para toda la vida. Madrid: Agencia Laín Entralgo; 2004. p. 192.

4. Barba-Téllez MN, Cuenca-Díaz M, Gómez AR. Piaget y L.S. Vigotsky en el análisis de la relación entre educación y desarrolo. Revista Iberoamericana de Educación 2007; 42/7: 1-12.

5. Domínguez-Castellano A, Cerro R, Bueno C, Bringas MJ, Balonga B, Royo JL. Factores predictivos de las in- 
fecciones nosocomiales producidas por microorganismos grampositivos y gramnegativos. Enferm Infecc Microbiol Clin 1995; 13: 19-27.

6. O’Neill M, Baxter CM. Evaluation of an integrated curriculum using problem-based learning in a clinical environment: the Manchester experience. Med Educ 2000; 34: 222-30.

7. Branda LA. El aprendizaje basado en problemas. De herejía artificial a res popularis. Educ Med 2009; 12: 11-23.

8. Fasce HE, Calderón BM, Braga IL, De Orúe RM, mayer SH, Wagemann BH, et al. Utilización del aprendizaje basado en problemas en la enseñanza de la física en estudiantes de medicina. Comparación con enseñanza tradicional. Rev Med Chile 2001; 129: 1031-7.
9. Albanese M, Mitchell S. Problem-based learning: a review of literature on its outcomes and implementation issues. Acad Med 1993; 68: 52-81.

10. Vernon D, Blake RL. Does problem based learning works. A meta-analysis of evaluative research. Acad Med 1993; 68: 550-63.

11. Schmidt HG, Vermeulen L, Van der Molen HT. Long-term effects of problem-based learning: a comparison of competencies acquired by graduates of a problem-based and a conventional medical school. Med Educ 2006; 40: 562-7.

12. Prince KJ, Van Eijs PW, Boshuizen HP, Van der Vleuten $\mathrm{CP}$, Scherpbier AJ. General competencies of problembased learning (PBL) and non-PBL graduates. Med Educ 2005; 39: 394-401. 